\title{
Activation Pattern During His-Bundle Pacing
}

Omar Yasin ${ }^{1}$, Jaime Bush ${ }^{2}$, Vaibhav Vaidya ${ }^{1}$, Jason Tri ${ }^{3}$, Martin van Zyl ${ }^{1}$, Peilin Xiao ${ }^{4}$, Jie $\operatorname{Han}^{5}$, Cory Scheuermann ${ }^{2}$, Samuel Asirvatham ${ }^{1}$, and Yong-Mei Cha ${ }^{1}$

${ }^{1}$ Mayo Clinic

${ }^{2}$ Boston Scientific Corp

${ }^{3}$ Mayo Clinic Minnesota

${ }^{4}$ The Second Affiliated Hospital of Chongqing Medical University

${ }^{5}$ Zhejiang University School of Medicine First Affiliated Hospital

July 11, 2021

Title: Activation Pattern During His-Bundle Pacing

Omar Yasin MD, MS ${ }^{1}$, Jamie Bush BS ${ }^{2}$, Vaibhav Vaidya MBBS ${ }^{1}$, Jason $\operatorname{Tri}^{1}$, Martin van Zyl, MBBCh ${ }^{1}$, Peilin Xiao $\mathrm{PhD}^{3}$, Jie Han MD, $\mathrm{PhD}^{4}$, Cory Scheuermann ${ }^{3}$, Samuel J. Asirvatham, MD ${ }^{1,5}$, Yong-Mei Cha, M.D ${ }^{1}$

1. Department of Cardiovascular Diseases, Mayo Clinic, Rochester, MN, USA

2. Boston Scientific Corporation, Washington DC, USA

3. The Second Affiliated Hospital of Chongqing Medical University, Chongqing China

4. The First Affiliated Hospital of Zhejiang University, Hangzhou, China

5. Department of Pediatrics and Adolescent Medicine, Mayo clinic, Rochester, MN, USA

Funding: None

Disclosures: None

Corresponding Author

Yong-Mei Cha, MD, FHRS

Department of Cardiovascular Diseases, Mayo Clinic

$2001^{\text {st }}$ ST SW

Rochester, MN 55901

Email:ycha@mayo.edu

\section{Introduction}

Biventricular pacing proved its efficacy in patients with wide QRS complex and reduced ejection fraction.(1) HB pacing may have similar benefits to biventricular pacing through restoration of interventricular synchrony.(2) To investigate this claim, we compared the activation pattern during HB pacing to that of sinus rhythm in canine animal model.

\section{Methods}

All procedures were conducted at Mayo Clinic, Rochester, MN after review and approval by the Institutional Animal Care and Use Committee (IACUC). Animals were fasted overnight, general anesthesia was induced using ketamine and diazepam and maintained with inhaled Isoflurane. 
Anatomic, voltage and activation maps were created using a 64-electrode INTELLAMAP ORION catheter and the RHYTHMIA HDx (Boston Scientific Corporation, Washington, DC) system. Right ventricular (RV) and left ventricular (LV) maps were created in sinus rhythm and during HB pacing. HB pacing was achieved using an active-fixation pacing lead (model 3830) and a deflectable sheath (model C304; Medtronic, Fidley, $\mathrm{MN})$. Pacing parameters ensured HB pacing by: 1) setting a rate faster than the animal's intrinsic heart rate, 2) adjusting output until an isoelectric interval was noted between pacing spike and QRS, 3) QRS duration less than 100ms on surface ECG with similar morphology to native QRS. Total LV and RV activation times were defined as the time difference between earliest and latest intra-cardiac signals in the corresponding chamber.

\section{Results}

LV and RV electro-anatomic maps were obtained at baseline and during $\mathrm{HB}$ pacing in four out of the five animals (one animal did not have RV map during HB pacing). Average QRS duration was $54 \pm 3.9 \mathrm{~ms}$ and $79 \pm 26.0 \mathrm{~ms}$ at baseline and during $\mathrm{HB}$ pacing respectively. $\mathrm{HV}$ interval at baseline was $28 \pm 6.3 \mathrm{~ms}$ and stim-V was $36 \pm 3.7 \mathrm{~ms}$ during $\mathrm{HB}$ pacing.

The earliest site of ventricular myocardial activation at baseline was in the LV mid-septum and was $5 \pm 1.9 \mathrm{~ms}$ earlier than the RV septum. During HB pacing, earliest site of ventricular activation was in the RV at the pacing site and $25 \pm 19.6 \mathrm{~ms}$ earlier than the LV. LV activation times were $63 \pm 6.9 \mathrm{~ms}$ at baseline and $75 \pm 25.6 \mathrm{~ms}$ during HB pacing. The activation sequence in the LV was mid-septum (earliest) followed by apex and then base during sinus rhythm, while during HB pacing, the earliest LV activation was in the high septum then spread down to the apex (Figure 1 ). On necropsy specimens, the HB pacing lead was found on the ventricular septum at or slightly below the membranous septum.

\section{Discussion}

We found a significant difference in the activation patterns between sinus rhythm and HB pacing. During sinus rhythm the earliest site of myocardial activation was in the LV septum and during HB pacing it was in the RV. The activation pattern of the LV was different in sinus rhythm (apex to base) when compared to HB (high septum to apex). Para-Hisian or non-selective HB pacing leads to simultaneous activation of the HB and adjacent myocardium.(3) This study suggests that during HB pacing, local myocardial capture can be present despite a narrow QRS and an isoelectric interval on surface ECG between pacing and earliest V.

1. Yancy CW, Jessup M, Bozkurt B et al. 2017 ACC/AHA/HFSA Focused Update of the 2013 ACCF/AHA Guideline for the Management of Heart Failure: A Report of the American College of Cardiology/American Heart Association Task Force on Clinical Practice Guidelines and the Heart Failure Society of America. J Am Coll Cardiol 2017;70:776-803.

2. Sharma PS, Dandamudi G, Herweg B et al. Permanent His-bundle pacing as an alternative to biventricular pacing for cardiac resynchronization therapy: A multicenter experience. Heart Rhythm 2018;15:413-420.

3. Cantu F, De Filippo P, Cardano P, De Luca A, Gavazzi A. Validation of criteria for selective his bundle and para-hisian permanent pacing. Pacing Clin Electrophysiol 2006;29:1326-33. 


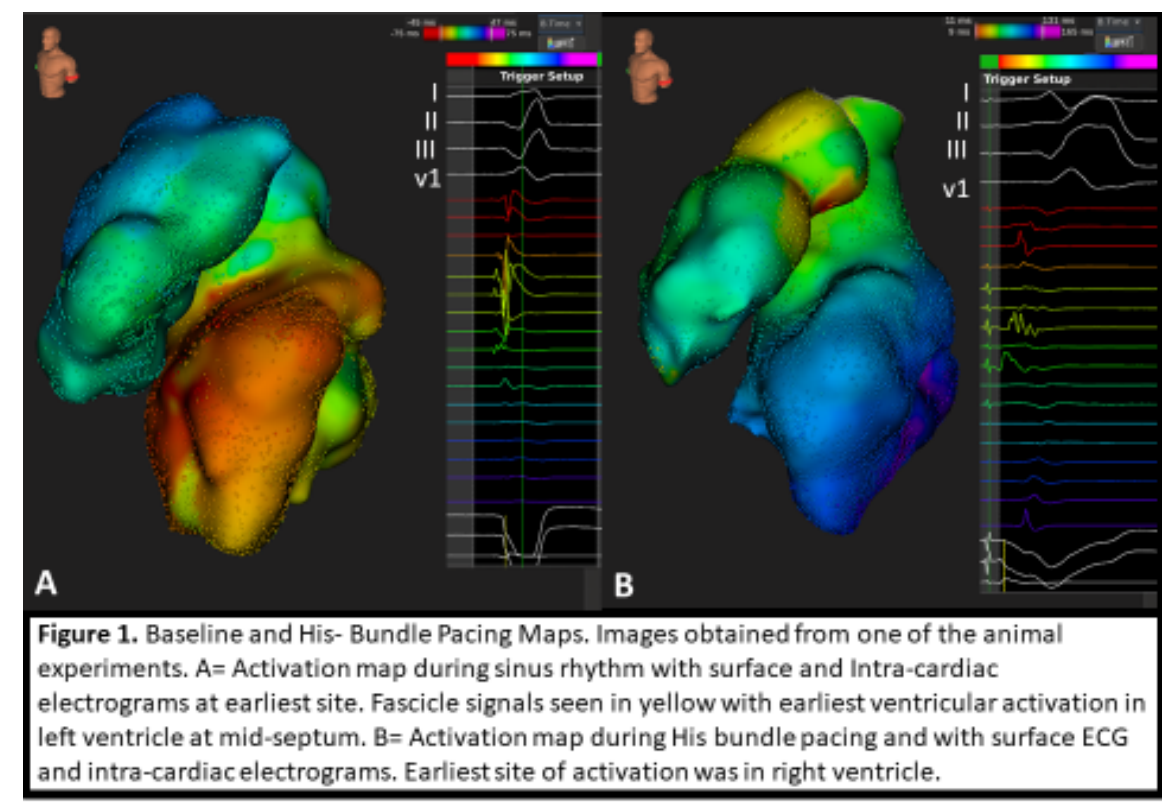

